\title{
Anthropologie, religion et modernité
}

Quelques réflexions sur le modernisme et le primitivisme des sciences de l'Homme

\section{Lionel Obadia}

\section{(2) OpenEdition}

\section{Journals}

Édition électronique

URL : https://journals.openedition.org/pa/1764

DOI : $10.4000 /$ pa.1764

ISSN : 2273-0362

Éditeur

Université Lumière Lyon 2

\section{Édition imprimée}

Date de publication : 1 janvier 2004

Pagination : 11-22

ISBN : 1634-7706

ISSN : 1634-7706

Référence électronique

Lionel Obadia, « Anthropologie, religion et modernité », Parcours anthropologiques [En ligne], 4 | 2004

mis en ligne le 06 juillet 2021, consulté le 21 juillet 2021. URL : http://journals.openedition.org/pa/

1764 ; DOI : https://doi.org/10.4000/pa.1764 


\title{
ANTHROPOLOGIE, RELIGION ET MODERNITÉ
}

\author{
Quelques réflexions sur le modernisme et le primitivisme \\ des sciences de l'Homme
}

\section{Prolégomènes}

Faisant écho à un mouvement de fond des sciences de l'homme, de nombreux anthropologues (surtout nord-américains) ont affirmé avoir engagé, depuis environ deux décennies, la discipline dans le tourbillon de la réflexivité et de la déconstruction postmoderne. Leurs sources d'inspiration sont désormais pleinement identifiées et ouvertement revendiquées: la fameuse French Theory (qui convoque, en vrac, des œuvres aussi variées que celles de Derrida, de Foucault, de Deleuze, de Baudrillard ou encore de Bourdieu), les Cultural Studies, de même que des approches critiques au sein même de l'anthropologie, comme celles de Geertz $^{1}$. Si l'on a le plus grand mal à identifier pleinement les tenants du postmodernisme, en revanche quantité d'auteurs américains (James Clifford, Michael Fischer, Georges Marcus pour n'en citer que quelques-uns parmi les plus connus) ou français (Christian Ghasarian) ont fondé leur analyse sur l'existence de ce courant d'idées et installé la déconstruction comme le fer de lance d'une perspective résolument "nouvelle» en anthropologie. On pourrait longuement s'interroger sur le caractère original de cette démarche, mais tel n'est pas ici le propos.

Ce qui se manifeste avec acuité à la lecture des sciences de l'Homme contemporaines, c'est que certains concepts semblent étonnamment résister au traitement déconstructionniste. Si l'anthropologie a passé au crible quantité de ses outils et de ses théories, la notion de "modernité " aurait sans doute autant mérité (sinon plus) que d'autres de subir le sort de la déconstruction. Pourtant, elle fait partie de ces référents qui présentent une étonnante résistance à ce type d'analyse. Une immunité qui laisse à penser que les territoires de la connaissance soumis à la salutaire mais périlleuse expérience d'une minutieuse critique épistémologique représentent de simples îlots sans jamais véritablement constituer un continent, pour reprendre la métaphore géographique utilisée, dans d'autres circonstances, par J.C. Passeron ${ }^{2}$. Une insularité qui révèle en outre les difficultés de l'entreprise, et les périls qui la guettent : voir se réduire l'anthropologie à une épistémologie ou à une méta-anthropologie est sans doute le plus dénoncé parce que le plus visible. Celui de voir se maintenir ou ressurgir des concrétions idéologiques ou culturelles (un peu rapidement frappées du sceau infamant des "prénotions" pour les positivistes) sous le vernis de concepts scientifiques en est un autre, qui semble à l'évidence regarder de près les débats autour de la modernité.

\section{De quelques usages courants de la modernité}

La première difficulté à se saisir d'une réflexion critique sur la modernité réside dans le sens même à conférer à un vocable d'une aussi large surface sémantique, d'ailleurs corrélative de son extension discursive ${ }^{3}$. La modernité revêt des sens différents selon les disciplines, les contextes de formulation, les conditions historiques ou encore les enjeux idéologiques qui se profilent en filigrane d'une critique ou d'une apologie. Trois disciplines, dont les régimes épistémologiques sont assez proches, sont en particulier concernées par la modernité : l'histoire, la sociologie et l'anthropologie. La modernité est d'abord essentielle pour et à l'histoire. Il n'est pas exclu de penser qu'elle en est la catégorie constitutive sans laquelle une certaine partie des historiens ne saurait mener leur réflexion. Si le point est des plus importants au plan méthodologique, c'est au plan théorique que les problèmes surgissent. Dans l'effort de mettre de l'ordre dans le flux chronologique des événements, les historiens ont en effet édifié la modernité comme une "phase " ou une " séquence» de l'histoire, selon un découpage dont par ailleurs l'extension temporelle fait l'objet de discussions. Ici, la modernité se définit comme référent de l'histoire qui localise les autres phases dans un enchaînement linéaire au regard duquel les séquences antérieures sont "pré " ou "proto" modernes. Subsumées au référent "modernité", d'autres séquences se retrouvent, dans une philosophie historique, inscrites dans sa continuité : "post ", "hyper " ou " ultra " modernes... la constellation des variantes s'organise autour de ce cœur "solaire" qu'est la modernité. Il en va de même pour la sociologie, même si les raisons qui président à ces choix sont liées à d'autres processus d'institutionnalisation et de fondation théorique. L'idée de modernité se retrouve également aux sources de la sociologie (dans l'œuvre des fondateurs Weber et Durkheim, notamment), pour en devenir, à l'occasion d'une formulation explicite, le pivot central qui en détermine l'objet, le terrain et les analyses (depuis Parsons et sa théorie de la « modernisation »). Parallèlement, il est courant que le vocable trouve sa place dans des réflexions anthropologiques, sans qu'il soit, là encore, toujours pleinement explicité ou qu'il fasse l'objet d'une analyse profonde. Au mieux, il réfère à un usage lâche, dans le cadre d'une définition nominale (conventionnelle), en opposition à une définition réelle qui, seule, examine avec précision les rapports entre les mots et les choses ${ }^{4}$ - pour paraphraser M. Foucault. 
Après avoir été confondu avec les vocables de " civilisation" ou de "progrès ", la modernité est surtout apparue en anthropologie sous la forme d'un couple l'unissant à la " tradition » (polarité qui continue d'être ancrée dans les mœurs de quantités de lieux de pensée pour les sciences sociales), longtemps affiché comme l'un des axes principaux de la réflexion. Les objets étaient ainsi traités sous l'angle d'un rapport entre deux états (" entre tradition et modernité ») ou dans la perspective d'un processus ("de la tradition à la modernité »). Cette polarité, relevant d'une distinction de nature ou de morphologie entre des sociétés ou des dynamiques historiques, a permis, en son temps et au miroir de la sociologie, de spécifier certains objets et terrains de l'anthropologie. Si la réflexion anthropologique s'est depuis quelques années consacrée à l'analyse des raisons présidant aux variations sémantiques des typologies sociétales, il n'avait échappé à personne que les sociétés "industrielles", "occidentales" et "modernes" semblent former un seul et même ensemble, de même que ces présumées «sociétés traditionnelles » qui s'étendent dans le temps et dans l'espace extra-occidental englobaient du même coup des humanités aussi variées qu'éloignées.

\section{Déconstruire la " modernité "}

Pour se prémunir de toute forme de réductionnisme lapidaire ou d'excès de naïveté force est de reconnaître que la modernité relève $a$ priori d'une pensée plus complexe de la société et de l'histoire que ce premier repérage disciplinaire le laisse penser. Elle ne saurait se limiter à des définitions lexicales enfermées dans des cadres conceptuels restreints, et convoque plutôt de larges et mobiles réseaux sémantiques. Les réflexions qui vont suivre ont pour vocation de remettre la modernité à sa place, c'est-à-dire à la restituer au domaine du discours (scientifique et idéologique), d'en considérer les usages, les contextes et les objets du monde qu'elle est supposée désigner. Ce ne sera pas la première fois que les catégories des sciences sociales et en particulier celles de l'anthropologie font l'objet d'une analyse critique : il suffit de mentionner les travaux d'Edmund Leach sur le mariage, ceux de Rodney Needham sur le concept de croyance, la controverse qui a opposé LéviStrauss à Radcliffe-Brown sur la notion de structure pour constater le caractère récurrent d'un tel examen.

Il est sans doute impossible à la pensée anthropologique de s'exercer sans avoir recours à des notions très générales telles que celle de modernité : néanmoins, les considérer comme des données de l'expérience (historique) revient à leur conférer une consistance dont elles ne bénéficient sans doute pas autant que la postérité du vocable le laisse croire. Sur la base de la lecture que Kuhn offre de l'histoire des sciences ${ }^{5}$, tout laisse à penser que la postérité du vocable modernité a résidé dans le caractère paradigmatique des thèses modernistes. En anthropologie, Georges Balandier a par exemple contribué - comme de nombreux autres - à produire une déconstruction réifiante en questionnant la modernité pour mieux lui conférer le double statut de contexte historique et de configuration sociologique, et l'inscrire dans une approche paradigmatique du présent ${ }^{6}$. Sa propre définition de la modernité s'avère en fait être avant tout une anti-définition : "le mouvement, plus l'incertitude ". Ces critères ont l'avantage et l'inconvénient d'être fondés sur une conception dynamique des sociétés humaines et de l'histoire, dont on se rappelle qu'elle avait, entre autres vocations, celle d'invalider les conceptions prétendument "fixistes ${ }^{7}$ de Lévi-Strauss ${ }^{8}$. Mais alors que Lévi-Strauss, contrairement à la démarche structuraliste qu'il affectionne tant, définissait les sociétés primitives par leur fonctionnement propre sans référer de manière systématique à leur opposé conceptuel, les sociétés modernes, Balandier assigne à la tradition un caractère culturellement contraignant reposant sur une certaine densité des relations sociales, alors qu'il installe, à leur opposé, des sociétés "modernes" qui en figurent l'image inversée, justifiant de la sorte une distinction paradoxale traçant une ligne de démarcation (fixe) entre des objets (démarqués) là où ses thèses prétendaient introduire l'image dynamique d'un continuum sociologique réversible.

De par le caractère universel qu'on a voulu lui attribuer, la modernité s'avère de plus inscrite dans une pensée catégorielle et générique, qui présente le double inconvénient d'une occultation de ses généalogies historiques et idéologiques, en l'occurrence les processus à partir desquels elle s'est constituée, et d'une autoréférentialité. Si l'anthropologie s'assigne précisément comme objet ce qui est de l'ordre de la variation ${ }^{9}$, les catégories génériques telles que celle de "modernité " font naturellement problème. Chercher à enfermer la modernité dans une définition générale consiste déjà à lui conférer une réalité que lui consacre en premier lieu le langage. Les éclairantes leçons du pragmatisme en linguistique et de la philosophie de la connaissance kantienne s'appliquent ici fort à propos : désigner la modernité c'est déjà lui donner une réalité, et en établir les critères revient à l'inscrire dans des cadres conceptuels prédéfinis qui se trouvent ainsi consolidés avant même d'avoir été confrontés à la réalité. La démarche de déconstruction procèderait, comme certains l'affirment, d'une lecture postmoderne de la modernité, adoptant ainsi une posture plus critique à l'endroit du réalisme épistémologique. Or, modernité et postmodernité ne sont rien d'autre, au niveau de l'analyse, que des construits intellectuels, des abstractions inscrites dans un langage qui leur confère une réalité. Si l'on suit les analyses de Passeron sur les rapports entre langage naturel et langage scientifique, on ne retiendra pas ici la différence habituellement tracée entre les deux courants d'idées : la modernité apparaît à l'évidence comme un récit voire comme un "mythe » pour les sociétés occidentales, le postmodernisme comme une version particulière de la mythologie occidentale contemporaine, légitimée par les œuvres de Jean-François Lyotard, de Jean Baudrillard ou encore de Michel Maffesoli. 


\section{Les critères flottants de la modernité}

Nombreux sont les auteurs qui se sont efforcés de fixer les critères de la modernité sur l'examen de traits morphologiques : formes de la transmission culturelle, de la nature et de la profondeur des consciences historiques, des formes du rapport au passé, des modes d'organisation sociale, des systèmes politiques... qui, dans la plupart des analyses, présentent un caractère organique et forment système. Parmi les conséquences de la modernité les plus couramment énoncées, la pluralisation ou le pluralisme occupent une place centrale. C'est dans cette première matrice que se trouvent les développements les plus conséquents d'une pensée linéaire qui part du postulat d'une unité initiale à une diversité finale, qu'elle se situe au plan social (théories de la différenciation) au plan culturel (théorie du multiculturalisme) ou au plan religieux (théorie du pluralisme des croyances). On retrouve en écho certaines théories du métissage ou de l'hybridation mais seulement celles qui stipulent l'existence d'un état primitif inaltéré et homogène antérieur à un état moderne foncièrement bigarré. En ouverture d'un ouvrage à vocation généraliste sur $L$ 'état des religions dans le monde, Paul Blanquart écrit :

«La modernité des années quatre-vingt est marquée par un phénomène à la fois double et unique : le développement considérable des techniques et moyens de communication et le brassage généralisé des populations qui, jusque-là, se répartissaient la planète en zones distinctes et relativement homogènes ${ }^{10}$. Il en résulte une liquéfaction des identités héritées, collectives et individuelles ${ }^{11}$ ».

La citation se passerait presque de commentaire : elle contient toute l'idéologie moderniste des sciences sociales actuelles, constituée sur l'idée que le monde est entré récemment « en modernité » et que celle-ci introduit de la diversité là où auparavant prédominait l'unité - validant ainsi une conception homogénéisante des aires culturelles et de la tradition, et une vision «fluide» et hétérogénéisante de la modernité, comme si la face d'un monde bien ordonné avait changé entièrement sous la pression d'un emballement incontrôlé de l'histoire. Ces modélisations évolutives (pour ne pas d'emblée dire "évolutionnistes ») se retrouvent à l'occasion dans les théories anthropologiques, lesquelles esquissent dans un bel élan commun un glissement des formes mouvantes des objets de l'anthropologie sur l'échelle historique qui mène "de la tradition à la postmodernité ", "de la culture à la multiculture " ou à la "polyculture ${ }^{12}$. Par un glissement rarement explicité, les transformations qui affectent le social et le culturel se retrouvent in extenso dans le domaine religieux, ce qui n'est pas sans poser quelques problèmes méthodologiques. Les transformations religieuses sont en effet le plus souvent rapportées à des conditions historiques générales (dont l'influence est certes manifeste sur les premières) sans que les liens de causalité entre les premières et les secondes soient toujours pleinement mis à jour: la théorisation des changements sociaux et religieux relève alors plus d'une posture axiomatique que d'une véritable démonstration des chaînes de causalités entre les deux ordres de phénomènes ${ }^{13}$.

\section{MODERNITÉ ET RELIGION}

Les débats qui se sont constitués autour de l'axe "religion et modernité " ont connu des développements plus massifs en sociologie qu'en anthropologie et, dans une perspective quelque peu différente, en histoire des religions. Il fallait s'attendre à ce que la littérature sociologique mette en critique la notion de "religion": mais on s'étonne qu'elle ne l'ait assez peu fait pour celle de "modernité ». Pour les sociologues, l'affaire est entendue : la modernité est à la fois contexte historique et configuration sociologique dont la réalité, la densité et la morphologie font l'objet d'un tel consensus, qu'il est peu utile de la mettre en question. "Une modernité qui, quand elle s'interroge sur le concept de religion, traite en fait d'elle-même" affirme pourtant en 2003 le sociologue Jean-Paul Willaime ${ }^{14}$ avant de se livrer à l'habituel inventaire critique des définitions de la religion - sans jamais, dans la même livraison, évoquer en serait-ce qu'une seule fois les paramètres de cette fameuse modernité qui se lirait en miroir de la religion. C'est au lecteur de reconstituer patiemment cette modernité à l'essence insaisissable, en collectant patiemment toutes ses manifestations (et elles sont nombreuses) dans le domaine religieux : quête, choix, liberté, mélange, distance critique, autonomisation, individualisme, rationalisation, crise, déclin, irréligion, athéisme, etc. Tout se passe comme si la modernité ne pouvait être mise en question lorsqu'elle interroge la religion : la première qui représente le cadre de référence à partir duquel se mesurent les transformations de la seconde, est en quelque sorte la variable indépendante de l'analyse - elle en représente donc un postulat ou une prémisse, mais en aucun cas l'objet de l'analyse. En sus de cette exemption axiomatique de la modernité, la religion apparaît comme l'unique théâtre de ses effets : la "sphère" de la religion serait ainsi affectée seule par la modernité. Ce qui suppose que la modernité est extérieure à la religion, ou qu'elle possède une identité ontologique de nature différente : elle est alors facteur, agent, force, influence, mais dans tous les cas, elle façonne les formes de la religion, dont le caractère passif et "soumis " continue à se trouver dans la posture d'une traditionalité. Et lorsque les religions revendiquent une opinion ou une attitude contradictoire à cette lecture moderniste de leur histoire, elles sont alors taxées de traditionnalisme ou de "repli " traditionaliste, qui touche en particulier - mais pas exclusivement - les religions extra-occidentales (islam, hindouisme). Ainsi, la génération actuelle des chercheurs (en France: J-P. Willaime, D. Hervieu-Léger, F. Champion ou A. Piette, en Grande Bretagne: G. Davie, B. Wilson, en Italie : M. Introvigne, en Belgique : J. Rémy, F. Houtard, en Suisse : R. Campiche...) ne fait pas secret d'une approche qui examine les effets de la modernité sur la religion - en l'occurrence, les effets d'une modernité occidentale sur des formes religieuses occidentales. 
Clichés inversés : le faux "primitivisme " de l'ethnologie, le vrai "modernisme" de la sociologie

Le peu d'exploration, en anthropologie, des questions qui intéressent tant les sociologues peuvent s'interpréter comme une différence d'objet, de perspective ou de méthode. Certes, anthropologie des religions et sociologie religieuse n'ont de cesse d'affirmer qu'elles communiquent et se fécondent mutuellement. Le silence des ethnologues s'expliquerait-il en vertu des limites géoculturelles (celles de l'Occident) dans lesquelles le débat " religion » et « modernité » prend corps et forme, contribuant ainsi à la reproduction d'un «Grand Partage " qui a longtemps écartés les anthropologues de tout débat sur leurs propres sociétés ? En demeurant écarté d'un débat où ils sont convoqués (bien malgré eux), le risque est grand pour les ethnologues de corroborer une topographie imaginaire des régions et des cultures qui distribue de manière différenciée les degrés ou la profondeur de l'inscription dans une modernité occidentalocentrée à travers le prisme de la religion.

On peut en outre se demander s'il n'existe pas une différence fondamentale entre la manière dont les ethnologues et les sociologues ont pensé et traité le 14 fait religieux. D'abord, il est vrai que les ethnologues se sont intéressés pour la plupart d'entre eux à des religions différentes de celles examinées par les sociologues : quoi qu'on en dise, le christianisme et, jusqu'à un certain point, les monothéismes occidentaux (j'y inclus l'islam) n'ont pas donné lieu à de véritables "anthropologies » à la manière du chamanisme, de l'animisme ou des grands polythéismes. À la rigueur, les acculturations non-occidentales des monothéismes apparaissent comme des variantes suffisamment exotiques pour avoir retenu l'attention des ethnologues. Sur un plan plus épistémologique, cette fois, on pensait que la profonde, justifiée et incisive critique que l'anthropologie a entreprise depuis plus de deux décennies, contre ses propres perspectives, concepts et méthodes, se serait étendue au-delà des frontières poreuses de la discipline. On aurait pu également croire que les assauts répétés contre le primitivisme ${ }^{15}$ et le relativisme allaient enfin affranchir, par effet de contagion, les sciences sociales de ces reliquats de l'évolutionnisme et d'un certain culturalisme. Mais, comme si elles répondaient à une disposition fondamentale à penser l'altérité ou à quelque stratégie de légitimation institutionnelle, ces tendances font montre d'une évidente endurance. Une anecdote récente, à mon sens particulièrement révélatrice, vient confirmer ce propos : à l'occasion d'une discussion avec un collègue, Directeur de recherches dans une grande institution scientifique française, le thème de la religion est venu naturellement dans la conversation. Relevant mon penchant pour cette question, le collègue se plaignait de son côté que les sociologues ne témoignaient qu'un intérêt tout relatif aux phénomènes religieux. Et de finir l'entretien sur cette phrase: "Ce n'est pas comme vous, en ethnologie: avec l'étude des sociétés primitives, vous êtes familier du religieux ». Les ethnologues seraient ainsi au contact d'une religion par excès transpirant de toutes part chez les "primitifs ", les sociologues étant quant à eux à la recherche d'une religion par défaut qu'il faut exhumer des recoins dérobés de nos sociétés modernes. Voilà qui ne manque pas de trancher singulièrement avec l'intense visibilité du religieux dans nos sociétés (intérêt intellectuel généralisé, questions des sectes et de la laïcité, succès des religions extrêmeorientales, présence perpétuelle des sociétés secrètes dans les arcanes du pouvoir, etc.).

Pour prendre un exemple supplémentaire, pioché (presque) au hasard dans la littérature scientifique récente, $\mathrm{P}$. Blanquart, déjà cité ici, écrit :

"Dans les sociétés traditionnelles, telles que nous les décrivent les ethnologues ${ }^{16}$, l'individu n'existait pas et les différentes activités constitutives de la vie sociale étaient encastrées les unes dans les autres. Cette étonnante expérience d'intégration et de cohérence était due au primat du symbolique [...]. En instituant le groupe [...] en enveloppant l'ensemble des activités tout en pénétrant chacune d'entre elles de l'intérieur, la religion lui assurait une identité stable. Une société (ou une civilisation) était alors définie par $u n e^{17}$ religion, fondement à la fois de sa cohésion interne et de sa distinction d'avec les autres ${ }^{18}$.

Les ethnologues seraient donc, pour les tenants d'une sociologie généraliste du fait religieux, pourvoyeurs de conceptions qui ratifient des conceptions éminemment primitivistes, plus ou moins directement héritées de la lecture des œuvres d'inspiration évolutionniste de Tylor ou de LévyBruhl. Les caractéristiques assignées à ces sociétés primitives reflètent ainsi, par miroir inversé, celles qui sont supposées caractériser en propre les sociétés modernes: cohésion, communauté et croyance s'opposent à anomie, individualisme, incrédulité. Une représentation plus réelle que la réalité qui contribue également à la construction imaginaire d'un "âge d'or » des civilisations et des religions perpétuellement rejeté dans une altérité temporelle et géographique (i.e. l'objet de l'anthropologie selon ses observateurs extérieurs) : c'est ainsi l'Occident qui se pense et pense le monde à travers ses propres catégories idéologiques.

\section{LA MODERNITÉ À L'ÉPREUVE DE L'ETHNOGRAPHIE}

La tentation est grande de maintenir la réflexion dans un cadre abstrait, et de poursuivre indéfiniment la discussion sur le plan des seules idées. Mais l'anthropologie - pas plus que les autres sciences de l'Homme - ne saurait se passer des faits et de leur analyse sans quoi elle encourt le risque de se confiner aux débats d'idées - voire à la confrontation de croyances (de chercheurs). Lorsque les "paramètres" de la modernité, analysés sur le plan abstrait des débats, sont confrontés aux réalités du terrain, ils ne résistent en définitive pas longtemps. Et puisque l'anthropologie implique de partir des faits ; ces réflexions liminaires imposent un détour par des terrains explorés. Sans faire de l'expérience ethnographique un argument d'autorité, dans les divers pays où il m'est arrivé de résider plus ou moins longuement à des fins d'enquête (la France, le Népal, le Mexique, Israël...) ou plus temporairement en simple visiteur (La Roumanie, la Pologne, la Belgique, la Suisse, la Grande-Bretagne, l'Islande...) j'ai toujours été très 
étonné de voir que si les pratiques religieuses différaient profondément dans la forme d'organisation sociale dans laquelle elles s'inscrivaient, dans les élaborations symboliques qu'elles suscitaient, les attitudes des acteurs sociaux n'illustraient jamais parfaitement cet homo religiosus imposé par les sciences du religieux. Tous ces pays sont de plus engagés dans des processus d'industrialisation, de développement technologique et font la part belle à la science et à ses dérivés intellectuels... et tous témoignent parallèlement de la présence d'un religieux palpable, mais sans variation particulière d'intensité, à l'exception de moments d'effervescence ou d'événements ritualisés codifiés dans les traditions locales. L'Islande, pays des elfes et du surnaturel ? En cherchant bien, je n'y trouvais que des contes et légendes, des formes architecturales particulières déjà rencontrées ailleurs (cairns) qui ne possédaient là aucune autre signification que le marquage d'un espace déambulatoire. Nulle part, dans les villages de l'Himalaya (terre "sacrée » par excellence dans la fantasmagorie orientaliste), les moines, ascètes et chamanes, ainsi que leurs audiences, ne se montraient plus «illuminés " ou «mystiques " que les Français ou Belges convertis au bouddhisme les uns et les autres examinant avec une attitude mêlée d'espoir et de scepticisme ce qu'ils pouvaient retirer de manière très concrète de leurs pratiques religieuses : bonheur, prestige, fortune, guérison... En territoire Seri, sur la côte pacifique du Mexique, les danses sacrées continuent de réunir les villages entiers, pour que s'y manifestent sans effusion spirituelle particulière, la convivialité ordinaire, et les conflits qui en découlent. Les jeunes Polonais et Israéliens, prétendument ancrés dans les traditions dominantes de leurs pays respectifs, affirment vouloir se détourner de la religion, tout en refusant de voir se dissoudre leurs référents culturels... fondamentalement religieux. Somme toute, la cartographie prévue des binômes tradition/religieux "plein » ou " authentique " et modernité/religieux « vide» ou "transformé " ne trouve pas là sa pleine confirmation. Il n'est sans doute pas inutile d'exhumer ici quelques travaux classiques de l'anthropologie, qui semblent confirmer que cette idée est déjà depuis longtemps admise de manière plus ou moins explicite.

\section{La réflexivité à l'épreuve : l'équivoque croyance des sorciers et chamanes}

L'une des caractéristiques attribuées à la modernité qui semble faire l'unanimité en sociologie réside dans une conception "réflexive " de l'acteur social. Les causes en sont régulièrement attribuées à l'émergence d'une " conscience historique » ou de " conscience de classe ", mais aussi et surtout d'une réflexivité critique à l'endroit des représentations, valeurs et croyances qui constituent les environnements idéels, directement issue d'un double processus de différenciation sociale et d'individualisation ${ }^{19}$. Dans le domaine religieux, le contingent d'auteurs convoqués pour décliner cette thèse de concert et avec autorité a fait les beaux jours de certains numéros des Archives des Sciences Sociales des Religions ${ }^{20}$. Une telle perspective signifie donc que les acteurs sociaux ont, dans la modernité, "quelque chose" de plus que ceux qui résident «encore » dans « la tradition ». C'est donc moins cette aptitude à la réflexivité comme caractéristique de "la modernité " que sa dénégation pour les individus des sociétés "traditionnelles » qui fait problème et qui procède, à mon avis, de deux réductions. La première tient dans une lecture et une appropriation pour le moins simplificatrice d'une notion issue de la psychologie : la conscience réflexive (la capacité de l'esprit de se penser lui-même en action) est une propriété supposée générale de l'esprit humain depuis Hegel (malgré la tendance de ce dernier à penser des sociétés «primitives»). Pourquoi certaines sociétés en seraient-elles dépourvues? En situant l'analyse sur un plan idéologique, une telle démarche confine à une assignation primitiviste digne de la pensée "prélogique " énoncée un temps par Lévy-Bruhl ${ }^{21}$, et signale bien la permanence d'un primitivisme dans la Social Theory qu'affectionnent tant de chercheurs. Sous cet angle, les interlocuteurs des anthropologues continuent d'être de simples "reflets» des traditions de leurs sociétés, aveuglément soumis aux systèmes de croyances de leurs propres sociétés.

Alors qu'une certaine sociologie et qu'une certaine histoire ont voulu voir, dans un schéma évolutif, la croyance (archaïque) comme une forme antérieure ou différente de la connaissance (moderne), ce qu'a amené l'ethnologie, c'est une interrogation sur les " croyances" indigènes incluant dans une même analyse la "connaissance " de ces indigènes ... et celle des observateurs. La conclusion révèle que trop souvent, encore, un modèle occidental est projeté sur d'autres formes de croyances : croyance (irrationnelle et subjective) opposée au savoir (rationnel et objectif). Mais la complexité des opérations mentales mobilisées dans les formes "primitives » de la pensée religieuse et notamment le totémisme, interdit de penser la pensée «traditionnelle» comme "simple». Et la résurgence permanente, dans l'histoire occidentale, de courants d'idées religieuses, mais aussi sorcellaires ou "surnaturelles ", en particulier aux $\mathrm{XIX}^{\mathrm{e}}$ et $\mathrm{XX}^{\mathrm{e}}$ siècles (en pleine "modernité ", donc) invite à penser que de mêmes dispositions à la pensée «irrationnelle» et «rationnelle» se retrouvent à parts égales en Occident et ailleurs, où se donnent à observer dans les comportements religieux une même tension entre "doute" et «foi »: Gérard Lenclud a montré que la seule variation constatée réside dans la légitimité de leur expression $^{22}$. Enfin, depuis les intuitions de Lévistrauss $^{23}$ approfondies par Dan Sperber, les croyances ne sont plus "irrationnelles" mais relèvent d'un dispositif mental tout aussi rationnel que celui qui préside à la connaissance. Une simple expérience ethnographique - si courte soit-elle permet de ramener l'indigène à un autre rang dans l'ordre de la pensée que celui d' idiot culturel ${ }^{24}$ incapable de penser de manière distanciée ses propres traditions et d'évaluer de manière critique la plausibilité des croyances qu'elles offrent. De l'histoire de Quesalid (exhumée par Boas et analysée par Lévi-Strauss), ce jeune indien qui raillait le pouvoir des sorciers de sa tribu (et des autres), avant de se trouver lui-même pris à son propre jeu en étant considéré comme un "puissant 
sorcier ", Lévi-Strauss tire comme leçon la puissance des conventions collectives dans la production sociale de la magie et de la sorcellerie ${ }^{25}$. Une autre lecture de ce récit invite à réfléchir sur la question de la croyance elle-même, dans les sociétés "traditionnelles » : le fait le plus frappant du récit est que Quesalid ne croit pas au pouvoir sorcier, dont il s'efforce de démontrer le caractère superficiel et pantonimique. Un «primitif » doué d'une capacité critique et réflexive incisive : voilà qui donne à réfléchir. "Soyons tous comme Quesalid!» clame Bertrand Hell en rapportant l'histoire du sceptique qui allait devenir sorcier malgré lui à la démarche exploratoire de l'ethno$\operatorname{logue}^{26}$. "Nous sommes déjà tous des Quesalid » eston tenté de répondre au regard des similitudes constatées entre les différentes attitudes témoignées à l'endroit de la religion, que l'on soit issu d'une société «traditionnelle » ou « moderne ${ }^{27}$.

\section{Distance et théâtralisation : le jeu théâtral du} rite sara

Les anthropologues ont également leur part de responsabilité dans la théorie du primat du symbolique et la prééminence des croyances sur la réflexivité. Les discours "ritologiques » modernes sont alors autant de questions posées à une conception symboliste du rite ${ }^{28}$ : sa présence est-elle concomitante de celle de la religion (dans le domaine politique, sportif ou ailleurs ) ou existe-t-il une autonomie des actes qualifiés de rituels par rapport à toute forme de croyances religieuses, comme dans la pensée de Goffman ${ }^{29}$ ? De LéviStrauss à Turner, en passant par Caillois ou Eliade, s'il y a des rites dans les sociétés étudiées par les ethnologues, alors ils sont logiquement porteurs de croyances. Mais une croyance (en tant qu'énoncé) ne se confond pas avec l'acte de croire - lui-même hautement problématique. L'existence d'un système de croyances normatives ne dit en définitive rien sur la nature de l'adhésion des individus à leurs rites et cultes. La fameuse anecdote de RadcliffeBrown est plus que jamais d'actualité : qui, de l'Asiatique et de l'Australien qui s'observent mutuellement offrir pour l'un, des aliments, pour l'autre, des fleurs à leurs défunts dans un cimetière, est celui qui "croit " que les morts ont encore de l'appétit ou qu'ils peuvent encore humer le parfum et contempler les couleurs des végétaux qu'on leur offre? Dans les sociétés que l'on qualifie encore actuellement de "traditionnelles", les individus participant à des rites religieux, sont-ils «dans " le rite et sont-ils convaincus de son efficacité ? Dans La mort Sara ${ }^{30}$, Robert Jaulin nous offre un éclairage ethnographique fort intéressant sur la question. Dans de très belles pages, l'ethnologue français décrit la mise en mouvement d'un rite d'initiation débutant par l'entrée en fanfare dans le village d'esprits anthropophages: les femmes, retirées dans les huttes avec les enfants, sont soustraites à la vue (périlleuses pour elles) des esprits (en fait les hommes déguisés). Pourtant, la curiosité les pousse à chercher confirmation (en tentant de braver l'interdit) de ce qu'elles savent pertinemment: la présence des hommes sous les masques, une information que ces derniers sont pour leur part peu enclins à laisser s'échapper (en renforçant la réclusion des femmes). Personne n'est dupe de la mise en scène et tout le jeu consiste à attribuer aux autres (les hommes aux femmes, les femmes aux enfants) des croyances dans ces manifestations surnaturelles envers lesquelles chacun se montre perplexe. Jaulin démontre admirablement que la ritualité procède ici largement d'une théâtralité qui doit sa permanence moins à de prétendues croyances effectives dans des esprits, que dans la savante orchestration d'un déroulement dramatique dont tous les acteurs et les spectateurs doivent scrupuleusement respecter les règles... et tentent de s'en affranchir. Une ritualité "traditionnelle» certes saturée de symbolique, mais nullement engluée dans une crédulité primitive qui, une fois encore, se sera dérobée à l'analyse.

\section{HISTOIRE, MYTHES ET RÉCITS}

On situera maintenant le propos sur un plan plus macroscopique, pour encore examiner les liens entre les différents plans de la réalité : celui de l'histoire et des structures.

\section{Modernité, sécularisation, évolutionnisme}

La pensée de la modernité interroge non seulement le statut des faits qui servent à l'établir comme théorie, mais également celui de l'histoire dont elle est dérivée, que la conception qui la sous-tend apparaisse clairement dans l'analyse ou en soit gommée. Les changements si souvent évoqués dans les «formes" des religions ou la "morphologies du sacré " supposent un changement à l'échelle des structures sociale et symbolique. Le problème qui se pose est celui des cadres de référence qui servent à cette démonstration. Les monothéismes, et plus particulièrement le catholicisme, ont longtemps fait (et continuent de faire) office de modèle-étalon à partir duquel sont mesurés les changements: différenciation, anomie, déstructuration, désinstitutionnalisation, déclin, décomposition, émiettement, dérégulation, rupture, fractionnement... l'étendue lexicale est loin de s'épuiser que, déjà, le caractère "destructeur " de la modernité se fait jour. Plus récemment, d'autres vocables sont venus contrebalancer ce diagnostic: renouveau, métamorphose, revival, recomposition, pluralisation, réinstitutionnalisation... Les thèses du changement religieux s'écrivent ainsi en termes de recul ou de déclin, et de renouveau ou de réveil. Ce modèle en "flux" et "en reflux " interroge ici de nouveau les conceptions historiques: est-ce une histoire circulaire qui passerait d'une phase de flux à une phase de reflux, une circularité qui n'est pas sans rappeler la structure de la temporalité mythique, ou une histoire linéaire dont les modernités changent au gré de leur positionnement sur l'échelle de l'évolution?

\section{Diffractions : de la communauté à la société}

Les lectures de l'histoire sociale et religieuse ne peuvent à tout le moins se défaire de ces antiques et désuets modèles sur lesquels continuent de reposer la grande majorité des analyses en sociologie et histoire des religions : ceux de l'évolutionnisme du $\mathrm{XIX}^{\mathrm{e}}$ siècle. Parce qu'elles prétendent se fonder sur une histoire générative aux séquences clairement définies qui s'enchaînent dans un parfait déroulement, et qu'elles supposent qu'il existe des 
modalités évolutives qui relient ces séquences les unes aux autres, l'histoire et la sociologie des religions (du moins celles qui sont ici désignées comme relevant de l'allégeance moderniste) s'inscrivent pleinement dans un raisonnement évolutionniste dont elles ont le plus grand mal à s'affranchir. Et pour cause : ce serait l'ensemble de leurs modélisations qui s'effondrerait si elles devaient les abandonner! Qu'on se rappelle simplement les efforts de Durkheim pour établir les formes élémentaires de la religion à partir de ses manifestations "primitives" (1912), mais aussi que le fameux sociologue américain Robert Bellah est l'auteur, en 1965, d'un modèle de l'évolution religieuse où se retrouvent des «stades" (primitif, archä̈que, historique, proto-moderne et enfin moderne $)^{31}$ ou que Roger Lapointe affirme sans faillir que « la dichotomie du moderne et du primitif demeure tout à fait pertinente pour l'étude de la société en général [...] elle laisse ouverte la question de savoir ce qu'ont été exactement [sic !] les sociétés anciennes ou modernes, aux points de vue économique, politique, artistique, religieux, etc. $»^{32}$, défendant une pensée évolutionniste qui pense résoudre la question de la singularité du religieux «moderne» par référence à des classifications historiques qui enferment, plus qu'elles ne révèlent, des formes religieuses singulières.

Les sociologues sont ainsi confrontés à des problèmes identiques à ceux qui se sont posés, il y a maintenant un siècle, aux anthropologues évolutionnistes. En premier lieu, la modernité (à l'époque, la «civilisation") est le fruit d'un processus évolutif : mais elle est également son point d'aboutissement et, dans ce sens, la perspective (dans le sens physique et idéologique du terme) qui sert à la lecture de l'histoire mériterait une prise de distance. Or, la modernité interroge l'histoire pour mieux lui servir de modèle de référence sans que cette même modernité soit ellemême interrogée, à la manière d'un Lewis Morgan qui aura subdivisé les stades "sauvage" et "barbare " de son histoire générale et leur aura associé quantité de propriétés singulières sans jamais questionner la nature du stade "civilisé " qui semble de la sorte prémuni contre toute forme de critique. Reste à savoir jusqu'à quel point l'anthropologie peut laisser s'installer dans la durée des idées qui lui sont attribuées et dans lesquelles elle ne se reconnaît plus depuis longtemps. Un modèle historique qui situerait la communauté à l'origine de la société s'inscrit dans une théorie de la différenciation: l'un des critères généralement attribués, depuis Durkheim, aux sociétés modernes - mais que l'on retrouve également dans toutes les sociologies d'inspiration wébérienne. Combien de temps ces fameuses communautés ethnologiques prétendument indifférenciées, c'est-à-dire cohésives, religieuses, "pleines » de symbolique serviront-elles de référence-miroir pour une modernité qui les a imaginées ? Jusqu'à quel point l'étude des religions restera-t-elle astreinte au théocentrisme et à l'ethnocentrisme de la perspective moderniste dont la pertinence autoproclamée procède d'une cécité comparative et d'un perspectivisme historique alors que les appels à la vigilance ont été lancés de toutes parts, par les anthropologues, préhistoriens (André Leroi-Gourhan) ou psychologues (Antoine Vergote)?

\section{Primitif/sacré, modernité/désenchantement}

Mais les sociologues se plaisent encore et toujours à voir dans les "sociétés archaïques" des normes sociales enserrées dans le sacré ${ }^{33}$. Dans la continuité de ces présupposés primitivistes, la théorie $d u$ "désenchantement du monde », formulée initialement par Weber et reprise par Marcel Gauchet $^{34}$, qui connaît actuellement une certaine postérité, apparaît comme une variante de la théorie de la sécularisation qui veut que la rationalisation progressive de "la » société entraîne au pire, le déclin de la sacralité et/ou de la religiosité, au mieux, son "déplacement "vers la sphère privée - et, partant, son exclusion d'une sphère publique où elle est censée s'être depuis longtemps installée (ce qui se traduit généralement en termes de "sortie de la religion»). Dans leur Esquisse d'une théorie générale de la magie Hubert et Mauss (1905) ont montré que l'opposition public - privé n'est pas le signe d'un émiettement récent de la religion mais sa polarisation antique autour de deux espaces complémentaires où se déploient conjointement la face « religieuse » et la face " magique " des croyances. Le très remarqué Religion and Globalization de Peter Beyer (1994) apporte de plus un démenti historique au "repli » de la religion à travers sa propagation à l'échelle des nations et d'un «système mondial " qui ne représente pas véritablement une "sphère privée ». La thèse du "désenchantement" appelle une thèse du "réenchantement». L'une comme l'autre sont fondées sur l'idée butoir à la pensée qu'un monde "prémoderne " était «enchanté ». Fondé sur la fiction romantique d'un monde peuplé d'esprits, de dieux et de farfadets, cette féerie archaïque relève d'un imaginaire de l'altérité désigné par Bryan Turner sous la formule générique d'Orientalisme ${ }^{35}$. Assistet-on réellement à l'exclusion du sacré, du mythe, de la foi dans les sociétés " modernes"? Ou au retour du sacré et du mythe sous des formes "nouvelles »? ou à un simple déplacement de la sacralité et de la pensée mythique sur d'autres objets?

L'archaïque au cœur du moderne : histoire linéaire et redondances structurelles

Les thèses $d u$ "déclin » et du « renouveau ", du "désenchantement" et du "réenchantement" s'épuisent à saisir un sens linéaire que l'histoire n'a sans doute pas. Même si peu d'auteurs d'y réfèrent (ou s'y réfèrent encore), l'antique débat qui a opposé Georges Balandier à Claude Lévi-Strauss sur leurs conceptions respectives de l'histoire semble plus que jamais résonner dans les controverses qui alimentent les débats sur la modernité et la religion. La grande majorité des auteurs (sociologues) penche pour une analyse fondée sur l'idée d'une production permanente et récurrente de formes religieuses inédites, et la littérature se trouve encombrée de ces "nouvelles " religions, " nouvelles" croyances, "nouvelles» expériences, « nouvelles" formes de sociabilité religieuse... Par delà cette lecture de l'histoire qui fait la part belle aux aspects les plus visibles (l'événementiel), d'autres, en se rappelant à la dialectique des permanences et des mutations, ont su discerner certaines régularités sous-jacentes à l'histoire, qui laissent à penser que cette dernière pourrait en définitive n'être le théâtre que d'un nombre limité 
de forces agissantes ou de processus, qui sont en outre indépendants d'un contexte historique singulier. Le sociologue James Beckford souligne par exemple dès 1983 que "the history of religion could be written as an unceasing struggle between the forces of institutionalization and disruption ", affranchissant ainsi (involontairement) l'analyse d'une trop manifeste association entre "déstructuration" des religions et modernités ${ }^{36}$. Le même Beckford semblait même prêt à rallier la cause des structuralistes en décelant une cocasse redondance de l'histoire au sein des fameux "nouveaux mouvements religieux ", ceux-là même qui sont supposés refléter au mieux l'innovation religieuse : "it is ironic " affirme-t-il dans le même ouvrage "that some movements inadvertently reproduce in the conversion experience of their own members, some of the most strongly abhorred features of the traditional religious systems $»^{37}$. Loin de nous l'idée de réfuter l'idée que l'histoire est faite de transformations et de turbulences: rien n'interdit néanmoins de s'interroger sur le niveau de la réalité auquel s'applique cette vision de l'histoire.

Dans une livraison récente de la revue Ethnologie française $^{38}$, la sociologue Françoise Champion affirme: "l'étude des nouveaux mouvements religieux n'avait, jusqu'à une époque très récente, que peu retenu l'attention des ethnologues; il semble bien que les choses soient en train de changer ». Malgré la proposition de la sociologue d'ouvrir aux ethnologues ce champ "inexploré " de l'innovation religieuse, une véritable anthropologie des "nouveaux mouvements religieux " n'a pas été établie comme un véritable programme de recherche, et à plus d'un titre, on peut s'interroger sur la pertinence d'un tel programme. Certes, Georges Balandier et Roger Bastide avaient déjà, dans des contextes culturels assez différents, largement contribué à la compréhension des transitions religieuses et des formes de culte syncrétique consécutives - comme les dits "nouveaux mouvements religieux " actuels - à des changements sociaux rapides. Mais les ethnologues sont habitués à explorer les sociétés - comme les historiens - en rapportant leur analyse à une perspective diachronique profonde, et les formes d'innovation religieuse leur apparaissent rarement dans cette perspective - quelque peu teintée de sensationnalisme - de la "déferlante " soudaine de religions «inédites ${ }^{39}$.

C'est avec la plus grande prudence et au regard d'une analyse fine des rapports entre religions existantes et des formes "nouvelles» que Marc Augé amène la question de la dialectique des permanences et des mutations, les dernières retenant manifestement bien plus que les premières l'attention des modernistes, les premières n'étant pas nécessairement, comme la posture primitiviste assignée aux ethnologues pourrait le faire croire, l'expression d'un attachement aveugle aux formes séculaires de la religion: "Comme les mutations brusques sont rares en histoire, comme une organisation sociale est rarement bouleversée d'un seul coup et tout entière, du fait d'une révolution interne ou d'une intervention extérieure, il n'est pas si étonnant que la cohérence idéologique puisse se maintenir alors même que l'ordre social est en fait atteint et perturbé. [...] On ne dira pas pour autant que les changements (les vicissitudes historiques) ne peuvent se repérer au plan idéologique [...] Seulement, le rapport entre la réalité matérielle et historique de la vie sociale et les représentations est fonctionnel, non spéculaire $»^{40}$.

Tout ceci nous ramène avec force vers l'idée que le cadre des "mutations" de la religion "en modernité " peut s'insérer dans une pensée historique structurelle non matricielle. La théorie de la sécularisation, actuellement encore au cœur des débats, n'admet pas, elle non plus, de lecture univoque : ni ses causes (sécularisation "interne" comme chez Dumont ${ }^{41}$, sécularisation "externe " comme chez les sociologues durkheimiens), ni son extension (elle affecte très différemment les milieux ruraux et urbains, les sociétés industrielles et agricoles, l'Occident et le reste du monde...) ni ses conséquences (disparition ou affaissement de $l a$ religion pour les plus pessimistes, métamorphose et renouveau pour les optimistes). Il n'en demeure pas moins que l'on ne peut que s'interroger sur la portée théorique de la sécularisation : ce «destin " de la religion n'est en définitive que celui de certaines formes (le christianisme d'abord, et, jusqu'à un certain point, le judaïsme) dans certains contextes (industriels et occidentaux). Comment pourrait-on expliquer dans cette perspective que d'autres religions n'aient pas connu le même sort historique lorsqu'elles ont été confrontées à des conditions identiques (le polythéisme hindou face à l'industrialisation de l'Inde) ou que tous les monothéismes n'aient pas donné lieu à une sécularisation (l'islam)?

Si pour certains cette sécularisation est purement et simplement émancipation de la religion (donc rupture), pour d'autres elle revêt les formes d'une continuité fondée sur la traduction dans des termes laïcs d'idéologies religieuses. Sous le niveau le plus manifeste des transformations religieuses, celui des événements ou des formes d'organisation idéologiques et sociales perceptibles, se situerait ainsi un niveau latent qui dévoilerait la permanence d'une forme "structurale " ou profonde de la religion: les idéologies du progrès et de la modernité ne seraient rien d'autre, sur un plan à la fois sémiotique, morphologique et sémantique, qu'une expression (qui ne dit pas son nom) des formes religieuses antérieures contre lesquelles elles se sont précisément établies ${ }^{42}$. Certains philosophes vont plus loin, en énonçant l'un des nombreux paradoxes de la modernité : la modernité se confond avec l'herméneutique, une forme de pensée rationaliste qui a désacralisé les propres mythes de la modernité (le progrès, l'objectivité), réintroduisant par là même les mythes religieux au cœur de la modernité - qui d'ailleurs s'en accommode très bien en retrouvant ainsi l'assise des structures symboliques sur lesquelles elle s'était constitué $e^{43}$. Loin des "mythologiques"de Barthes (en fait, des lectures sémiotiques de la vie ordinaire), Mircéa Eliade s'était lui-même amusé à déceler (en les trouvant) les formes structurelles des mythologies antiques dans les expressions idéologiques du XX $\mathrm{X}^{\mathrm{e}}$ siècle ${ }^{44}$. 


\section{MODERNITÉ ET CULTURE, MODERNITÉ CULTURELLE}

Concluons cette série de remarques (loin de couvrir l'ensemble des questions soulevées au sein du débat " modernité et religion ") pour rétablir un semblant de perspective anthropologique. On peut tout à loisir explorer les déterminants idéologiques du concept de modernité ou la portée de son opérationnalité. Mais ce détour par l'épistémologie nous ramène finalement au problème premier et toujours essentiel, celui de la nature de la modernité et sa place dans le raisonnement: déconstruire ne peut être une entreprise salutaire pour les Sciences de l'Homme qu'à compter du moment où celle-ci s'accompagne d'une alternative constructive pour la recherche.

\section{Catégorie indigène ou scientifique ?}

L'anthropologie ne peut faire l'économie d'une analyse détaillée et critique de la modernité. Une première réponse, d'inspiration réaliste, se trouve dans l'œuvre de Marc Augé : la modernité existe et elle infléchit les rapports sociaux de telle manière qu'il s'avère nécessaire de repenser la culture ellemême : la totalité réifiée disparaît au profit d'une production dynamique des interactions sociales ${ }^{45}$. Si les conclusions auxquelles Augé est conduit sont d'ordre méthodologique, on est en droit de se demander si elles ne pouvaient pas dériver de l'analyse d'autres conditions historiques voire de n'importe quelles autres conditions historiques que celles qui caractérisent la contemporanéité théorisée comme une modernité ${ }^{46}$. Le problème vient du constat, qu'il faudra bien faire un jour, que la notion de modernité, comme lecture métahistorique de l'histoire, métaculturelle des transformations culturelles, suprasociale des changements sociaux... n'est en définitive pas universellement applicable. Pour Bruno Latour, la modernité n'existe tout simplement pas : les modes de pensée " primitifs " et " modernes » qui sont régis par des mécanismes identiques produisent des objets identiques ${ }^{47}$. Il n'en demeure pas moins que la catégorie "modernité " continue d'être largement utilisée comme opérateur conceptuel dans les sciences de l'Homme. Les anthropologues nordaméricains ont poussé l'analyse dans un sens différent: si la modernité est un terme occidental qui a vocation à devenir concept anthropologique, celui-ci doit être confronté à chaque terrain pour mieux être redéfini. Les critères prétendument universels de la modernité et l'extension de ses conséquences sont alors examinés pour mieux mettre en critique les aspects ethnocentrés d'une telle catégorisation conceptuelle, et prendre sérieusement au sérieux les dynamiques culturelles locales d'absorption de la modernité occidentale ${ }^{48}$. Cette inflexion vers la «pluralisation» de la modernitét ${ }^{49}$ se traduit dans le domaine religieux par l'introduction récente du concept de multiples modernities, forgé par Robert W. Hefner pour rendre compte des stratégies différenciées des religions face à ce qui apparaît comme leurs propres défis historiques ${ }^{50}$. La modernité est ainsi une réalité, mais formulée à sa manière pour chaque religion, selon ce qui apparaît à ses adeptes comme relevant de l'évolution historique et de l'adaptation (ou la résistance) à un contexte global.

\section{Une conception historicisée de la modernité}

Encore une fois, ce qui fait problème, c'est ici moins la notion de modernité que sa généralité ou son extension à des cadres d'analyse qui transcendent le contexte localisé dans lequel elle était auparavant signifiante. La conversion d'échelle d'un local, certes, étendu à un vaste espace géoculturel (l'Occident), à l'universel (le monde) auquel elle peut prétendre sous les traits d'un concept anthropologique n'offre en définitive qu'une alternative : soit la modernité est suprasociale et métahistorique par essence et dans ce cas sa réification sous la forme d'un vocable transculturel est tout à fait légitime ; soit elle est résolument ancrée dans la société et dans la culture et dans ce cas elle réfère à une modélisation de l'histoire qu'il convient d'expliciter, en rencontrant tous les problèmes préalablement exposés, et bien d'autres.

Le problème de la notion de modernité, comme l'a montré Danilo Martucelli dans le contexte de la sociologie, c'est qu'elle prend corps et est formulée dans le cadre d'une pensée historique de l'histoire qui occulte sa propre historicité ${ }^{51}$. En ce sens, les prétentions des modernistes à en faire un vocable conceptuel métaculturel semblent s'amenuiser à mesure que ses éléments constitutifs en révèlent l'ancrage culturel. Vytavtas Kavolis a bien montré qu'il s'agissait là d'une irréductible circularité puisque, selon lui, la modernité ne peut être comprise qu'à travers le prisme de la culture moderne $^{52}$. Elle n'est donc qu'une catégorie sémantique culturelle superposée à la compréhension de processus de changement qui ne sont signifiants qu'à partir de ce cadre de référence, et qui nous informent utilement sur les orientations théoriques de la recherche en un temps donné : la quête des origines qui traverse de part en part les sciences religieuses du XIX siècle s'est transmuée, au $\mathrm{XX}^{\mathrm{e}}$ siècle, en frénésie pour le futur de religion ${ }^{53}$.

À chaque période ses modes et ses marottes inscrites dans les idéologies de l'air du temps. La notion de "crise", par exemple, ne laisse pas d'étonner. Récurrente dans les discours actuels, elle revêt sous l'apparence d'actualité une profondeur historique et des manifestations itératives, qui signalent d'ailleurs bien ce qu'elle est censée occulter: en l'occurrence l'extension d'une focale culturelle (la crise du christianisme et/ou de la civilisation occidentale chez les philosophes du $\mathrm{XIX}^{\mathrm{e}}$ siècle et les penseurs de la première moitié du $\mathrm{XX}^{\mathrm{e}}$ tels René Guénon), à une perspective universelle (crise de la société "mondiale ", de «la» religion en général, etc.). Mais les religions sontelles vraiment en "crise" ailleurs que dans le discours de ceux qui défendent cette thèse? Un simple regard rétrospectif sur les vicissitudes historiques des grands monothéismes comme des systèmes polythéistes montre de manière éclatante que l'équilibre est l'exception, la perturbation la règle. 
Une conception "culturelle" et "diffusionniste » de la modernité

La tentation est grande, nous l'avons vu, d'inscrire cette modélisation dans un raisonnement évolutionniste : celui de la discontinuité entre le primitif et le moderne, d'une différenciation sociale et d'un individualisme offerts aux seules sociétés occidentales, d'un découpage séquentiel et irréversible de l'histoire, qui fonde une tradition intellectuelle presque centenaire, depuis Lewis Morgan jusqu'à Ernst Gellner. Mais la littérature ethnologique et historique fournit des objections si nombreuses à cette stéréotypisation du «primitif » et du "moderne" que le modèle ne peut que s'effondrer sur lui-même. La modélisation historique semble alors se dérober éternellement à l'analyse, réconciliant un passé/primitif avec un présent/moderne qui en a toujours été constitutif : "from the start, then, it may be that "modernity" existed a long time ago " selon Alan MacFarlane ${ }^{54}$ alors que pour Vytvatas Kavolis "modernizing" trends are as ancient as civilization itself $»^{55}$.

Nombreux sont ceux qui, depuis Weber, n'ont de cesse de le répéter : la modernité est un produit de l'histoire occidentale. Si l'on maintient l'analyse au strict plan des données de l'histoire, elle est donc éminemment culturelle. Sur quelles bases a-t-elle pu de facto être considérée comme universelle ? Les postures convergentes de Augé et de Balandier font de la modernité un produit de l'histoire occidentale tout en lui conférant le statut de précipité métahistoricisé. Si l'on repose ici la question de l'applicabilité de la modernité et des problèmes que pose son universalité, reste à savoir comment elle a été érigée au rang d' "état» ou de "condition " universelle. Nulle explication fondée sur des faits précis éclairant l'extensivité de la modernité (et plus encore de la post, ultra, hyper ou surmodernité) ne sera donnée du côté de la Social Theory ou des sociophilosophies: l'opération de généralisation ou d'universalisation semble bien n'être que de nature strictement intellectuelle.

La modernité est donc bien plus universaliste qu'universelle, et n'est universalisée que par l'intermédiaire de processus de diffusion ou d'extension identifiables, en l'occurrence, des processus de mondialisation $^{56}$, ce qui autorise par exemple Anthony Giddens à confondre purement et simplement « modernité » et « mondialisation ${ }^{57}$. La modernité culturelle est alors singulière : soit elle s'inscrit dans une lecture diffusionniste des influences occidentales sur le reste du monde, soit la lecture de ce qui se déroule hors de l'Occident procède d'une pure et simple projection ethnocentrée des catégories occidentales sur le reste du monde. L'exemple le plus frappant est celui de la sécularisation (un avatar de la modernité) dont la prétendue universalité a autorisé certains chercheurs à penser la définition de la religion et les transformations qui l'affectent sur la seule base de référence des vicissitudes historiques des monothéismes occidentaux, et plus particulièrement du christianisme, et donc à penser l'extension de ces transformations à l'échelle de toutes les religions sur la simple base du caractère universel, non pas des phénomènes mais des catégories conceptuelles pour les désigner. Certes, la mondialisation des religions (avant l'heure de "la" mondialisation) a amené de profondes transformations à l'échelle de la planète, de même que l'expansion des modèles idéologiques séculiers de l'Occident via la colonisation et l'économie de marché. Mais sans défendre une position trop relativiste (qui reviendrait à réintroduire du culturalisme dans l'analyse), la prudence (et les travaux récents la confirment) veut que l'on examine précisément dans chaque contexte culturel et social local, les effets de ces mondialisations, leurs influences et leurs acculturations. Ce qui justifie, à mes yeux, une approche diffusionniste de la modernité.

\section{Paradoxes de la prédiction, prudences anticipatoires}

En fin de parcours, il n'est sans doute pas inutile de revenir sur les circonvolutions paradoxales du débat " religion et modernité ». Il est pour le moins étonnant que les plus fervents hérauts des thèses modernistes - celles de l'avènement d'un homo religiosus rationalis ou modernus se substituant à un homo religiosus traditionalis - ont parfois été les mêmes qui invitaient au discernement entre une modélisation abstraite de la réalité et une réalité que la pondération scientifique aurait amené à théoriser dans sa complexité. Mircéa Eliade est l'un de ceux-là, lorsqu'il affirme, en 1968 :

\begin{abstract}
«"L'Homme total" n'est jamais complètement désacralisé, et on est en droit de douter qu'une désacralisation totale soit possible. La sécularisation remporte un beau succès au niveau de la vie consciente [...] mais aucun homme normal ne peut être réduit à son activité consciente, rationnelle car l'Homme moderne n'a pas cessé de rêver [...] il ne vit pas seulement dans un monde historique et naturel, mais aussi dans un monde existentiel, privé et dans un univers imaginaire $»^{58}$.
\end{abstract}

Ainsi, les "crises" et "renouveaux», "décomposition" et " recomposition ", "déclin " et " retour ", "flux » et " reflux » du religieux entre autres dichotomies qui émaillent le vocabulaire des défenseurs d'une thèse moderniste (qu'elle soit d'inspiration séculariste ou ultramoderniste) apparaissent-ils en dernière instance comme les représentations formulées par une communauté intellectuelle (composée en grande partie d'universitaires). Dans son stimulant ouvrage The persistence of religion, le trop peu lu révérend Andrew M. Greeley en avait fait un point de départ de sa critique des analyses contemporaines de la religion, dénonçant le décalage entre le discours des spécialistes et la réalité ${ }^{59}$. Si l'on rejoint ici sur de nombreux points Greeley, nous sommes ici tentés de relativiser le propos pour mieux prévenir la controverse, et de le situer dans une perspective ethnologique. Sans nier l'existence de processus de changements religieux, le réalisme positiviste n'est à l'évidence plus approprié à leur compréhension. Avec Modernity at large ${ }^{60}$ Arjun Appadurai avait ouvert la voie à la compréhension du rôle des imaginaires collectifs dans la construction idéologique du monde moderne. Dans son ouvrage Globalization, l'anthropologue américain nous invite à incorporer dans l'analyse les imaginaires de la recherche ${ }^{61}$. Plus qu'un filtre à une pensée de l'« exactitude ", c'est bien un imaginaire culturel qui 
médiatise le rapport à la réalité et modélise les rapports entre un passé, un présent et un futur construits ou reconstruits dans des cadres de pensée modernistes qui ont trouvé un terreau fertile au sein de l'université. Ces narrations de la religion et de l'histoire, dans l'histoire ou hors de l'histoire, les représentations de la religion ellemême, du changement et de la pérennité s'inscrivent à nos yeux dans un véritable Weltanschauung. Son caractère culturel se fait plus patent à mesure qu'il se diffuse dans d'autres corps sociaux et/ou contextes idéologiques que ceux de sa formulation initiale, et qu'il se trouve approprié par des acteurs individuels ou institutionnels qui construisent collectivement l'image mouvante d'une modernité qui, à n'en pas douter, changera encore de contenu et de forme au gré des changements impulsés par l'histoire.

\section{NOTES}

Qui, lui aussi, ne se reconnaît pas entièrement dans la paternité "postmoderne " qu'on confère encore à son œuvre. Cf. C. Geertz, Ici et Là-bas. L'Anthropologue comme auteur, Paris, Métailié, 1996, traduit de l'anglais par D. Lemoine.

2 Jean-Claude Passeron, Le raisonnement sociologique. L'espace non-poppérien du raisonnement naturel, Paris, Nathan, 1991.

3 Cf. Alexis Nouss, La modernité, Paris, P.U.F, 1995.

4 Cf. Melford E. Spiro, "La religion : problèmes de définition et d'explication", in Bradbury, R.E. et alii, Essais d'anthropologie religieuse, Paris, Gallimard, 1972 (1966), p.p. 85-125.

Thomas S. Kuhn, La structure des révolutions scientifiques, Paris, Flammarion, 1972.

Ce que la lecture de son ouvrage Le Détour (Paris, Fayard, 1985) révèle sans ambiguïté.

Admirablement résumées dans son article : Tradition, conformité, historicité, in L'Autre et l'ailleurs, hommage à Roger Bastide, s.l., Berger-Levrault, 1976, p.p. 15-37.

8 Enoncées dans les désormais " classiques "Entretiens avec Claude Lévi-Strauss (Georges Charbonnier, Paris, PlonJulliard, 1961).

$9 \quad$ On rejoindra dans ce sens la posture (paradoxale) de Lévi-Strauss qui, à la recherche des dimensions universelles de la pensée humaine, énonce comme objet de l'anthropologie (la culture) moins l'universel que la variation (Anthropologie structurale, Paris, Plon, 1974, passim).

10 C'est nous qui soulignons.

11 Paul Blanquart, "Le religieux : un nouvel enjeu stratégique », in Michel Clévenot (Ss la dir. de) L'état des religions dans le monde, Paris, La Découverte / Le cerf, 1987, p. 13.

12 Voir : De la tradition à la postmodernité, hommage à Jean Poirier, Paris, P.U.F, 1996.

13 Comme l'affirme courageusement le sociologue Bryan Wilson, Contemporary transformations of religion, London, Oxford University Press, 1976

14 Jean-Paul Willaime, "La religion : un lien social articulé au don ", Revue du Mauss, ${ }^{\circ} 22,2^{\mathrm{e}}$ semestre 2003, p. 259.

15 Notamment depuis Adam Kuper, The invention of primitive society: transformations of an illusion, London, Routledge, 1988.

Encore une fois souligné par nous.

Trois termes soulignés par nous.

Op. cit., p.p. 13-14.

Martucelli, Danilo, Sociologies de la modernité, Paris, Gallimard, Folio, 1999.

En particulier le $\mathrm{n}^{\circ} 81$ (mars 1993) précisément consacré à ce thème.

À propos duquel on oublie de mentionner que ce même auteur, encore trop souvent stigmatisé pour primitivisme outrancier, l'a d'ailleurs abandonnée à la fin de sa vie.

22 Gérard Lenclud, "Vues de l'esprit, art de l'autre. L'ethnologie et les croyances en pays de savoir ", Terrain, $\mathrm{n}^{\circ} 14$, mars $^{2}$ 1990 , p.p. $5-19$

Claude Lévi-Strauss, La pensée sauvage, Paris, Plon, 1962.

Selon l'expression de l'ethnométhodolgue Garkinkel, formulée dans un autre contexte théorique.

« Le sorcier et sa magie », in Anthropologie structurale, Paris, Plon, 1974, p.p. 191-212.

In Possession et Chamanisme. Les maîtres du désordre, Paris, Flammarion, 1999, p. 275.

Je me permets de citer ici un court ouvrage dans lequel j'ai déjà eu l'occasion de présenter des données qui vont dans le sens de cette démonstration. Lionel Obadia, La religion, Paris, Le Cavalier Bleu, 2004.

Victor W. Turner, Le phénomène rituel, Paris, Le Seuil, 1982.

Erwin Goffman, Les rites d'interaction, Paris, Editions de Minuit, 1974.

Robert Jaulin, La mort Sara. L'ordre de la vie ou la pensée de la mort au Tchad, Paris, Plon, 1971.

«Religious Evolution ", in W.A. Lessa, E. Z. Vogt (Eds), Reader in Comparative Religion, New York, Harper \& Row, 1965, cité par Roger Lapointe, Socio-anthropologie du religieux. Le cercle enchanté de la croyance, Genève, Librairie Droz, 1989 , p. 49.

Lapointe, op. cit., p. 48

Edgar Morin, Sociologie, Paris, Fayard, 1984, p. 431.

Marcel Gauchet, Le désenchantement du monde. Une histoire politique de la religion, Paris, Gallimard, 1985.

Turner, Bryan S., "Cosmopolitan Virtue : On Religion in a Global Age », European Journal of Social Theory, 4/2, 2001, p.p. 131-152.

36 Dans son introduction à : James A. Beckford (Ed), New Religious Movements and Rapid Social Change, Beverly Hills, Sage / Unesco, 1986, p. xi.

$37 \quad$ Id. p.xiii.

38 «La religion à l'épreuve des Nouveaux Mouvements religieux ", Les Nouveaux Mouvements Religieux, Ethnologie Française, 2000/4. 
Notion de «déferlante " que l'on retrouve entre autres sous la plume de Danièle Hervieu-Léger : « La religion des Européens. modernité, religion, sécularisation ", in G. Davie, Danièle Hervieu-Léger (Eds), Identités religieuses en Europe, Paris, La Découverte, 1996, p.p. 9-23.

40 Marc Augé, "Dieux et rituels ou rituels sans dieux? ", in John Middleton (Ed.), Anthropologie religieuse : les dieux et les rites, textes fondamentaux, Paris, Larousse, 1974., p. 35.

41 Louis Dumont, Essais sur l'individualisme, une perspective anthropologie sur l'idéologie moderne, Paris, Le Seuil, 1983.

42 Voir l'imparable démonstration de Pierre-André Taguieff, Du Progrès : biographie d'une utopie moderne, Paris, Librio, 2001.

43 Gianni Vattino, Au-delà de l'interprétation. La signification de l'herméneutique pour la philosophie, Paris, Bruxelles, De Boeck Université, 1997 (traduit de l'italien par Malou Somville-Garant), p.p. 49-62.

$44 \quad$ Mircea Eliade, Mythes, rêves et mystères, Gallimard, Paris, 1957.

$45 \quad$ Marc Augé, Le sens des autres. Actualités de l'anthropologie, Paris, Fayard, 1994.

$46 \quad$ Marc Augé, Pour une anthropologie des mondes contemporains, Paris, Aubier, 1994.

47 Bruno Latour, Nous n'avons jamais été modernes. Essai d'anthropologie symétrique, Paris, La découverte, 1994.

48 Englund, Harri, Leach, James, "Ethnography and the Meta-Narratives of Modernity ", Current Anthropology, vol. 41, 2, april 2000, p.p. 225-248.

49 Joel S. Kahn, «Anthropology and Modernity ", Current Anthropology, vol. 42, n 5, december 2001, p.p. 651-680.

50 Robert W. Hefner, "Multiples modernities : Christianity, Islam and Hinduism in a Globalizing Age ", Annual Review in Anthropology, 27, 1998, p.p. 83-104.

51 Sociologies de la modernité, op. cit.

52 Vytavtas Kavolis, "Nationalism, Modernization and the Polylogue of Civilizations ", Contemporary Civilization Review, $\mathrm{n}^{\circ} 25$ (Fall), 1991, p. 131.

$53 \quad$ Cf. Mircea Eliade, La nostalgie des origines, Paris, Gallimard, 1971.

54 Alan MacFarlane, "Ernst Gellner and the Escape to Modernity ", in John A. Hall, I.C. Jarvie (Eds), Transition to Modernity. Essays on Power, Wealth and Belief, s.d., Cambridge, Cambridge University Press, p. 131.

$55 \quad$ Op. cit., p. 124

Dans The condition of Postmodernity (Oxford, Blackwell, 1990) David Harvey contourne par exemple l'écueil de l'essentialisme de la «condition postmoderne " en la restituant à ses inscriptions sociologiques, économiques et politiques. Néanmoins, l'auteur cède encore une fois à la tentation d'en faire un produit réifié, métaculturel et supra social.

57 Anthony Giddens, Les conséquences de la modernité, Paris, L'Harmattan, 1994.

$22 \quad 58 \quad$ Dans la préface à l'édition anglaise de La nostalgie des origines, op. cit., p. 14.

59 Andrew M. Greeley, The persistence of religion, London, SCM Press Ltd, 1973, p.p. 3-4.

60 Modernity at Large: Cultural Dimensions of Globalization, Minneapolis, University of Minnesota Press, 1996.

61 "Grassroots Globalization and the Research Imagination ", in Arjun Appadurai (Ed.), Globalization, Durham \& London, Duke University Press, 2001, p.p. 1-21. 